\title{
Cognitive biometrics: a novel approach to person authentication
}

\author{
Kenneth Revett \\ British University in Egypt, \\ Faculty of Informatics \& Computer Science, \\ El-Sherouk City, Egypt \\ Email: ken.revett@bue.edu.eg
}

Biographical notes: Kenneth Revett is currently actively engaged in research focused in the development of the field of cognitive biometrics, which relies on the deployment of biosignals (EEG, ECG, EDR) for person authentication. $\mathrm{He}$ published the first textbook dedicated to Behavioral Biometrics (Wiley \& Sons) and is currently finishing up texts in the areas of cognitive robotics, bioinformatics for computer scientists, and cognitive biometrics. He is the editor of two Inderscience journals: Int. J. of Cognitive Biometrics and the Int. $J$. of Cognitive Performance Support. He has authored over 130 papers (journal and conference), and served on $30+$ international programme committees.

\section{Introduction}

Cognitive biometrics is a novel approach to user authentication and/or identification that utilises the response(s) of nervous tissue. The approach relies on the presentation of one or more stimuli, and the subsequent response(s) are acquired and used for authentication - a typical stimulus-response paradigm. The stimulus could be the presentation of a familiar photograph, a song, or a Rorschach ink blot, either individually or in various combinations. The stimulus is designed to induce a specific and reproducible change(s) in the (or a set of) state(s) of the individual. These reproducible changes can be recorded by a variety of methods - which currently include the electroencephalogram (EEG), the electrocardiogram (ECG), the electrodermal response (EDR), blood pulse volume (BVP), near-infrared spectroscopy (NIR), electromyogram (EMG), eye trackers (pupilometry), hemoencephalography (HEG), and related technologies. As with other forms of biometrics, issues such as reproducibility and uniqueness of the stimulus-response mappings must be acquired experimentally in order to quantify the reliability of a novel biometric paradigm. In the case of cognitive biometrics, the responses are changes in the (neuro)physiology of the subject, which are embedded in a continuous flow of ongoing dynamics (cognitive and/or physiological). The stimulus must elicit a response within this ongoing stream of activity that is both detectable and reliable. Further, the approach is constrained temporally (a few seconds is a suitable authentication time frame) and the technology must not be overly obtrusive (a wearable or lie detector test set-up is not practical nor acceptable).

The motivation for this approach is to provide a more intuitive and arguably a more robust and user-friendly authentication protocol that is suitable for both static and continuous authentication requirements. In addition, the cognitive approach can be 
combined with physiological approaches such as keystroke/mouse dynamics for example, augmenting the feature space and providing a truly multi-modal approach. Since cognitive biometrics deploys a much broader range of inputs for authentication (visual, auditory, olfactory, or any combination), a much larger variety of authentication approaches can be deployed. For instance, a user can be authenticated while playing a game for a short interval as opposed to entering their user ID and password. In addition, the future of human-computer interaction (HCI) may very well deploy a much wider range of interaction methodologies - such as speech, hand gestures, facial expressions, all of which possess some measure of the emotional and/or cognitive state of the user. Further, they tap into the same aspects of human behaviour that cognitive biometrics utilises - providing a natural link with a cognitive biometrics based mode of authentication (implementable over a range of computing platforms).

Cognitive biometrics has the daunting task of providing evidence to the relevant community at large that it is a viable alternative to anatomical and behavioural biometrics. It must be demonstrated that it provides robust classification accuracies (exceeding 95\%), without expensive and costly hardware, and must be readily accepted by the user community. In addition, the approach must be deployable in both a static and continuous fashion for maximal utility. The purpose of the International Journal of Cognitive Biometrics is to provide a forum for researchers to present their results to the relevant public at large. This inaugural issue presents several papers that focus on a wide variety of issues associated with implementing cognitive biometrics - focusing on factors such as acquirability, persistence, generalisability, and deployment issues. A brief description of each of the papers in this issue will be discussed, followed by what the editorial board members believe is an appropriate direction for this approach to follow in order to become successful.

\section{Paper summaries}

Nakayama and Abe's paper investigated the feasibility of classifying characters (Kanji characters) viewed by subjects (native Japanese) using single-trial Electroencephalogram (EEG) waveforms of the frontal and occipital areas of the brain (total of 21 electrodes, referenced to the ear lobe). This is a classic example of a lexical analysis task. As a training data set, event-related potentials (ERP) were created for each character from the first 20 trials, and the remainder was assigned to a test data set. To extract features of waveforms, a regression relationship between EEG and ERP waveforms was calculated from the training data set using the Support Vector Regression technique. Feature vectors for each trial were created as EEG waveforms from 100 to $800 \mathrm{msec}$ after stimulus presentation. The classification performance, measured as cross-validation rates (fivefold) of the test data set for each channel, increased incrementally during the perceptual process when the transformed waveforms were used. In addition, the performance rates increased when the classification was conducted using a combination of multiple electrodes located in the same area of the brain. These results provide evidence of a correlation between single trial EEG and ERPs in the context of Kanji character detection. Furthermore, the correlation is strengthened when multiple electrodes from the same cortical region are acquired simultaneously. 
The principle results from this study can be summarised as follows:

1 that single trial EEG recordings, when recorded either at the $\mathrm{Oz}$ (at $300 \mathrm{~ms}$ ) or $\mathrm{Fz}$ (at $500 \mathrm{~ms}$ ) electrodes are able to provide information regarding the nature of the stimulus (in this case either a proper Kanji symbol or not using a single channel

2 increasing the density of channels either in the frontal or occipital poles enhanced the classification accuracy, within the same time scales as the single channel results (300 and $500 \mathrm{~ms}$ respectively for $\mathrm{Oz}$ and $\mathrm{Fz}$ )

3 that regression analysis between ERPs and single channel EEG provides a mechanism for reducing the amount of data required for performance of a lexical analysis task.

More generally, these results indicate that a relatively short sampling time is required to provide reliable ERP data - in this case, 40 trials (each lasting $2 \mathrm{~s}$ on average) was sufficient for training, with testing occurring at the single trial level. The information content of both the single trial EEG and resulting training set derived ERP data were enhanced through the SVR technique. In addition, the results demonstrated that the time required for maximal correlation was lower for Oz electrodes (@300 ms) versus Fz electrodes (@500), consistent with the role these two brain regions have in a visual lexical analysis task.

Gupta, Palaniappan and Paramesran investigated the deployment of the P300 paradigm in terms of its suitability as a cognitive biometrics. Specifically, they investigated the role of distracters (via the popular Rapid Serial Visual Paradigm; RSVP) and stimulus position effects (via a specially designed a Spatially Varying Paradigm) on the reproducibility of latency and amplitude features of the P300. The investigation was carried out using both male and female subjects (aged 22-30) under conditions appropriate for a typical BCI based P300 experimental set-up. A four-category (the letters ' $A$ ', 'B', C', and 'D') were presented to the subjects in one of three conditions: basic odd-ball paradigm, the RSVP, and the Spatially Varying Paradigm. The tasks required users to focus visual attention at selected portions of the screen and make note of the occurrence of certain letters ('A-D'), and to make a mental count of the number of times a particular letter appeared. In the standard oddball paradigm, characters would appear at fixed locations and an eye-tracker could record the gaze of the subject and potentially replicate the actions of a valid user. To counteract this scenario, the stimuli were presented at various spatial locations, with the same frequency as the standard oddball approach. This would render eye tracking much less effective. In addition, the authors also investigated temporal disparities as an alternative approach to eye tracking hacking. In this scenario, the presentations were maintained at fixed locations, but would be presented at random time intervals, between which the subject would typically change their gaze slightly. The results of this experiment demonstrate that the temporal approach (RSVP) was the most effective strategy to counteract shoulder surfing and its variants (eye tracking). The system produced 100\% accuracy across all eight subjects with the RSVP approach, which was slightly higher than both the standard odd-ball and the Spatially Varying Paradigm.

In the paper by Israel and Irvine, the deployment of ECG was utilised as a biometric. This paper surveys much of the current ECG-based biometrics literature, emphasising the role(s) of factors that influence the reliability of ECG in terms of its usage as a biometric paradigm. The authors explore both external factors (environmental and sensor variants) 
as well as subject factors such as their emotional state, health, and related issues. The studies surveyed in this paper suggest that accuracies of close to $100 \%$ can be achieved with cooperative subjects. There are several factors that affect the reliability of an ECGbased approach that the authors highlight:

1 temporal stability (months to years after enrolment)

2 how the affective state of the subject influences the classification task

3 scalability of the approach.

The temporal stability of a subject's heartbeat (ECG signal) will potentially be influenced by a variety of factors - such as state of health and/or the affective state, i.e. whether they feel anxious or depressed for instance. This is the central issue that requires addressing within the cognitive biometrics community - quantifying how the affective and/or cognitive state (in EEG-based biometrics in particular) influences the underlying biosignal (ECG/EEG/and others such as the EDR. The temporal stability and generalisability issues are also key issues in both behavioural (i.e. keystroke dynamics) and cognitive biometrics, which is addressed in the paper by Tantawi et al. (this issue).

Tantawi and colleagues investigated the temporal stability and generalisability of the PhysioNet ECG data repository. This data repository contains ECG subject records from well over 100 individuals, with a variety of medical conditions, recorded over 1 day to several years apart. The paper describes a typical ECG processing pipeline on datasets acquired from PhysioNet, investigating the overall generalisability of this data repository, as well as addressing issues such as scalability. The first step in ECG processing is feature extraction, and the authors deployed a fiducial based approach, where visually apparent features such as the three principal modes (P, QRS, and $\mathrm{T}$ waves) are automatically extracted. These features are quantified and used for classification purposes in a supervised training methodology. In order to evaluate the generalisability of the dataset, records from one of the four data sets was used for training, and tested on records selected randomly from the other three data sets. This process was repeated such that each data set provided training samples, and the resulting classifier was tested on records selected from all the remaining data sets. These results generated classification accuracies on the order of $100 \%$ for subject recognition, and slightly lower values for heart beat recognition ( $83 \%$ in 1 or 2 instances, $100 \%$ for the remaining). This is a significant step towards establishing a schema for evaluating the generalisability of a biometric. Typically, many ECG-based biometric studies (or biometrics studies generally) are developed from small subject trials (5-20 subjects). Further, the testing phase is typically performed on the same data (records are held back for testing purposes) - but few apply their trained system to completely novel data sets. That the classification results were relatively independent of the training/testing data sets indicates that the approach used in this paper possesses significant generalisability. Furthermore, many of the records were test-retest cases, with intervals ranging from $<24$ hours (multiple recordings on the same day) or more than 1 year apart. This provides the opportunity to evaluate the developed ECG-based biometric schema in terms of temporal stability. The classifier was trained using records selected randomly, and tested on records selected from either the same day ( $\leq 24$ hours) or from records acquired several months to years apart (and vice versa). The subject classification accuracy was stable and did not suffer from the lack of temporal correlation, though there was a slight degradation in the heartbeat recognition accuracy. These results suggest that the schema developed in this 
work did possess generalisability and temporal stability required for deployment in a realistic manner. That is, subjects would not be required to re-enrol at a frequency that would be considered obtrusive.

\section{Discussion}

These papers provide examples of successful implementations of the cognitive biometrics approach. Even though they focused on ECG and EEG, there is no reason why the same basic methodology could not be applied to other biosignals such as EDR (the basis of the classic lie detector test), blood pulse volume (BVP), which provides a scaled down version of ECG data, near infrared spectroscopy (which can provide information regarding blood flow and oxygen saturation), pupilometry, and hemoencephalography (which combines EEG and NIR). There are two aspects that need addressing when presenting evidence of a 'cognitive biometric': one that the system is actually and purposely utilising cognitive aspects of individuals and the other, that the approach fits within the 'biometrics' domain. The former question will be addressed at the end, and attention is now turned to the 'biometric' component. To qualify as a biometric, most authors state that the following requirements must be met: (a) acquirability, (b) uniqueness, (c) persistence, and (d) must be difficult to spoof. Without going into significant detail, these issues will be addressed in order.

The technology exists to acquire a wide variety of biosignals - the first ECG was developed in the late 1890s, the first human EEG was reported in 1929, while Féré and Tarchanoff developed the scientific basis for the galvanic skin response (Tarchanoff's 'psychogalvanic reflex') (Féré, 1888; Tarchanoff, 1890; Berger, 1929). The technology has developed over the past century, largely driven by the needs of the medical and psychological communities. With only a slight modification, the technology can be tailored to suit the biometrics community directly, driven by market needs. This is indeed happening as we speak - a cursory search on the internet will reveal several companies actively developing dry EEG electrodes - which are designed to work without conductive gels and skin preparations. In addition, the number of electrodes can be reduced from that required for quantitative analysis as is the rule in QEEG approaches. As the studies in this issue have indicated, virtually perfect classification accuracy can be achieved with a single electrode. The same basic processes have been at work with ECG - which do not require 12-lead systems. In our lab, a basic 3-electrode system, placed on the wrists, is more than sufficient to develop a highly accurate person authentication system. As such systems find their way into the entertainment industry (computer/ console games) - there is no doubt that the technology will be tailored to small footprint and convenient implementations.

That everyone has a working heart and functioning brain is without doubt - so acquirability is typically not an issue - providing the technology is suitable in terms of user acceptance. In our studies, subjects state that the attachments of ECG electrodes on the wrist, EMG on the face, and GSR electrodes on the palmar surfaces are not overly restrictive and annoying, though this may be subject dependent. Overall, acquirability is simply a matter of convenience rather than biological plausibility.

Uniqueness is an issue that needs addressing - and is probably the most reported reason for non-cognitive biometric practitioners to reject this approach. When discussing cognitive biometrics in the context of more traditional strategies such as finger prints and 
iris scanners, colleagues state that the ECG is not a true biometric because of its inherent variability. For instance, if someone is stressed, their ECG changes and hence does not possess the constancy required in biometrics. That this is true is without doubt - there is variability in the ECG. It is known to vary with the emotional state of a subject. But, like a fingerprint, which typically requires some form of affine transformation prior to the application of the matching algorithm, cognitive biometrics makes the claim that the same process applies to an ECG record. What is required is a careful analysis of the impact emotion plays on the ECG recording - an area that is lacking in the literature. Clearly, stimuli can be produced that elicits a particular emotional state. If the ECG is recorded across a variety of emotional states, changes in the ECG record associated with each emotional state can be quantified and compared with a 'control' state record. If the differences can be quantified according to an appropriate feature space, then one could actually estimate the emotional state of the subject. Further, the impact of emotional state could be removed from the record, leaving the 'normal' ECG, performing the equivalent of an affine transformation. The same basic strategy holds for EDR and possibly related technologies such as NIR and BVP.

Although most studies deploying EEG as a means of user identification for example have found ample dimensionality in the data stream, a heretofore unexplored aspect of biosignals may be discoverable by applying the 'endophenotype' concept. This term was introduced in the early 1970s to describe a discrete genetically transmittable feature that is associated with a particular phenotype (disease states or clinical condition such as schizophrenia or ADHD). The key feature is that there is a heritable genotypic feature that is associated with a given phenotype - that is transmissible via a Mendelian type of mechanism, which is statistically more prevalent in genetically related individuals than in the general public (Gottesman and Shields, 1972; Gottesman and Gould, 2003; de Geus, 2010; Smit et al., 2010). A variety of endophenotypes have been identified: P50 prepulse inhibition, the latency and amplitude of the P300 ERP, smooth-pursuit eye movement (SPEM), certain aspects of working memory tasks, and a variety of other features of virtually all recordable biosignals possess a significant degree of heritability (upwards of 90\%) (Hanson et al., 1989; van Beijsterveldt et al., 1996; Anokhin et al., 2001; Wright et al., 2001; Smit et al., 2005; Hall et al., 2006; Chorlian et al., 2007; Zietsch et al., 2007; Wan et al., 2008). A rational approach to the feature space of say an EEG-based biometric is to produce stimuli that would extract a response that has a significant genetic component to it (e.g. P300 amplitude and/or latency, EEG coherence). There are a variety of genetically determined aspects of EEG, ECG, and EDR that could be exploited to enhance the uniqueness of the feature space (Russell et al., 1998; Crider et al., 2004; Newton-Cheh et al., 2005; Crider, 2008). This is a major area of research that needs to be addressed - and will require the collective efforts from a variety of disciplines.

Persistence refers to the temporal availability of a biometric feature, and is closely related to constancy - so both will be addressed as a single entity. The results from the study by Tantawi et al. (in this issue) indicate that the ECG appears to be stable over a period of at least 1 year. Whether or not this is an upper limit is an empirical question that remains to be settled. The temporal stability of EEG was investigated by Gupta et al. (in this issue), and their results demonstrate that aspects of EEG persists for 3 months, provided 2-3 electrodes are deployed. Again, whether this is an upper bound remains to be determined. By persistence is implied constancy - that a feature yields the same basic quantitative footprint over time. The results in the literature suggest that many of the 
features deployed in cognitive biometrics are persistent. We would argue that if the features were selected based on a genetic endowment basis, the persistence (and constancy) would extend to rational limits suitable for a biometric approach. This is a major research area that needs to be explored fully before final decisions in this regard can be made with certainty - but clearly the results indicate that this is not a deterrent.

Lastly, resistance to spoofing and related attacks is an issue that all biometric systems must be aware of. Clearly, the liveliness test is passed by a cognitive biometric approach (e.g. EEG, ECG, EDR, etc.) Could someone acquire a person's ECG and play it back? Certainly, but to counter this attack, the stimulus must be designed with considerable variation in mind. As mentioned previously, the wealth of stimuli suitable for cognitive biometrics provides a wealth of authentication schemes - game playing, listening to music, short video clips, as well as more traditional behavioural biometric approaches provide virtually an infinite amount of input stimuli for use as an authentication scheme. This holds for both static and continuous authentication modes - though the later provides many more opportunities to validate the user under a wide variety of stimulus challenges. This is one of the major advantages of the cognitive approach compared to anatomical biometrics such as finger prints and retinal scans. Further, this approach may suit more closely future person-computer interaction schema that may attempt to minimise traditional input devices such as keyboards and mice. As Julia Thorpe and colleagues proposed in 2005 - authenticating with our minds might be a reality in the near future - and certainly emotion based interactive gaming is already here (Thorpe et al., 2005).

In summary, cognitive biometrics is a novel approach to user authentication and/or identification that relies on the cognitive and affective responses of users, which are acquired via biosignal acquisition and psychological testing paradigms. Provided the proper stimuli are presented, the stimulus-response paradigm provides a powerful methodology for evaluating the authenticity of the subject requesting authentication. The concept of the endophenotype is central in this approach - as it provides a scientific basis for selecting stimuli, designed to elicit responses that have high heritability.

The next issue of the International Journal of Cognitive Biometrics will focus on the deployment of the EEG, one of the most heritable aspects of human physiology, as a means of user authentication. Pursuant to this aim, we seek to form a collaboration with the cognitivists and clinical geneticists, behavioural geneticists, amongst other disciplines, who are working from both ends towards the middle to produce a holistic approach to defining the interrelationships between cognition/affect and the underlying (patho)physiology (see de Geus and Boomsma, 2001 for a broader discussion of this approach). The cognitive biometrics community on the other hand is seeking cognitive/affective aspects of human physiology that are unique. The advantage this community provides is that experiments are performed within a scenario that directly determines the uniqueness of the feature(s). Most quantitative genetics studies are performed within well controlled and restrictive conditions, such as large MZ and DZ twin cohorts and possible singletons, reared together or apart (Russell et al., 1998; Crider et al., 2004; Newton-Cheh et al., 2005; Chorlian et al., 2007). This is required in order to quantify environmental and epigenetic factors. Ultimately though, the real test is to determine whether the identified features (endophenotypes) hold up in a large-scale examination, involving potentially tens of thousands of randomly selected subjects. Only then will the actual heritability of the feature(s) be explored sufficiently in order to render reliable estimates of heritability. Further, these types of studies in the context of 
biometrics may provide evidence of heretofore unknown endophenotypes. This is a clear case for a symbiotic relationship here that can be developed which will inform both communities. We at the International Journal of Cognitive Biometrics strongly encourage researchers in all relevant domains to submit work that demonstrates that there is a clear path for collaboration and help to instantiate cognitive biometrics as a truly innovative and future minded approach to computer security.

\section{References}

Anokhin, A.P., van Baal, G.C.M., van Beijsterveldt, C.E.M., De Geus, E.J.C., Grant, J. and Boomsma, D.I. (2001) 'Genetic correlation between the P300 event-related brain potential and the EEG power spectrum', Behavior Genetics, Vol. 31, No. 6, pp.545-554.

Berger, H. (1929) 'Über das Elektroenkephalogramm des Menschen', Archiv für Psychiatrie und Nervenkrankheiten, Vol. 87, pp.527-570.

Chorlian, D.B., Tang, Y., Rangaswamy, M., O'Connor, S., Rohrbaugh, J., Taylor, R. and Projesz, B. (2007) 'Heritability of EEG coherence in a large sib-pair population', Biological Psychiatry, Vol. 75, pp.260-266.

Crider, A. (2008) 'Personality and electrodermal response lability: an interpretation', Applied Psychophysiology and Biofeedback, Vol. 33, pp.141-148.

Crider, A., Kremen, W.S., Xian, H., Jacobson, K.C., Waterman, B., Eisen, S.A., Tsuang, M.T. and Lyons, M.J. (2004) 'Stability, consistency, and heritability of electrodermal response lability in middle-aged male twins', Psychophysiology, Vol. 41, No. 4, pp.501-509.

De Geus, E.J.C. (2010) 'From genotype to EEG endophenotype: a route for post-genomic understanding of complex psychiatric disease', Genome Medicine, Vol. 2, pp.63-66.

De Geus, E.J.C. and Boomsma, D.I. (2001) ‘A genetic neuroscience approach to human cognition', European Psychologist, Vol. 6, No. 4, pp.241-253.

Féré, C. (1888) 'Notes on changes in electrical resistance under the effect of sensory stimulation and emotion', Comptes Rendus des Seances de la Societe de Biologie (Ser 9), Vol. 5, pp.217-219.

Gottesman, I.I. and Shields, J. (1972) Schizophrenia and Genetics: A Twin Study Vantage Point, Academic Press, New York.

Gottesman, I.I. and Gould, T.D. (2003) 'The endophenotype concept in psychiatry: etymology and strategic intentions', American Journal of Psychiatry, Vol. 160, No. 4, pp.636-645.

Hall, M.H., Schulze, K., Rijsdijk, F., Picchioni, M., Ettinger, U., Bramon, E., Freedman, R., Murray, R.M. and Sham, P. (2006) 'Heritability and reliability of P300, P50 and duration mismatch negativity', Behavior Genetics, Vol. 36, pp.845-857.

Hanson, B., Tuna, N. and Bouchard, T. (1989) 'Genetic factors in the electrocardiograms of twins reared apart and together', American Journal of Cardiology, Vol. 63, pp.606-609.

Newton-Cheh, C., Larson, M.G. and Corey, D.C. (2005) 'QT interval is a heritable quantitative trait with evidence of linkage to chromosome 3 in a genome-wide linkage analysis: the Framingham Heart Study', Heart Rhythm, Vol. 2, pp.277-284.

Russell, M.W., Law, I., Sholinsky, P. and Fabsitz, R.R. (1998) 'Heritability of ECG measurements in adult male twins', Journal of Electrocardiology, Vol. 30, pp.64-68.

Smit, D.J.A., Posthuma, D., Boomsma, D.I. and De Geus, E.J.C. (2005) 'Heritability of background EEG across the power spectrum', Psychophysiology, Vol. 42, pp.691-697.

Smit, D.J.A., Boersma, M., van Beijsterveldt, C.E.M., Posthuma, D., Boomsma, D.I., Stam, C.J. and de Geus, E.J.C. (2010) 'Endophenotypes in a dynamically connected brain', Behavior Genetics, Vol. 40, pp.167-177.

Tarchanoff, J. (1890) 'Ueber die galvanischen Erscheinungen in der Haut des Menschen bei Reizungen der Sinnesorgane und beiverschiedenen Formen der psychischen Thatigkeit', Pflugers Arch. ges. Physiol., Vol. 46, pp.46-55. 
Thorpe, J., van Oorschot, P.C. and Somayaji, A. (2005) 'Pass-thoughts: authenticating with our minds', Paper presented in the Proceedings of the ACSA 2005 New Security Paradigms Workshop, September 2005, Lake Arrowhead, California, USA.

van Beijsterveldt, C.E.M., Molenaar, P.C.M., de Geus, E.J.C. and Boomsma, D.I. (1996) 'Heritability of human brain functioning as assessed by electroencephalography', American Journal of Genetics, Vol. 58, pp.562-573.

Wan, L., Friedman, B.H., Boutros, N.N. and Crawford, H.J. (2008) 'P50 sensory gating and attentional performance', International Journal of Psychophysiology, Vol. 67, pp.91-100.

Wright, M.J., Hansell, N.K., Geffen, G.M., Geffen, L.B., Smith, G.A. and Martin, N.G. (2001) 'Genetic influence on the variance in P3 amplitude', Behavior Genetics, Vol. 31, No. 6, pp.555-565.

Zietsch, B.P., Hansen, J.L., Hansell, N.K., Geffen, G.M., Martin, N.G. and Wright, M.J. (2007) 'Common and specific genetic influences on EEG power bands delta, theta, alpha, and beta', Biological Psychology, Vol. 75, pp.154-164. 